\title{
Seasonal variation of ocean bottom pressure derived from Gravity Recovery and Climate Experiment (GRACE): Local validation and global patterns
}

\author{
Torsten Kanzow, ${ }^{1}$ Frank Flechtner, ${ }^{2}$ Alan Chave, ${ }^{3}$ Roland Schmidt, ${ }^{2}$ Peter Schwintzer, ${ }^{2,4}$ \\ and Uwe Send ${ }^{1}$
}

Received 18 October 2004; revised 1 March 2005; accepted 13 April 2005; published 2 September 2005.

[1] The Gravity Recovery and Climate Experiment (GRACE) processing centers at the GeoForschungsZentrum Potsdam (GFZ) and the University of Texas Center for Space Research (UTCSR) provide time series of monthly gravity field solutions covering the period since mission launch in March 2002. Although the achieved accuracy still remains an order of magnitude below the mission's baseline goal, these time series have successfully been used to study terrestrial phenomena such as water storage variations. Over the oceans, the monthly gravity field solutions can be converted into estimates of the fluctuating ocean bottom pressure (OBP), which is the sum of atmospheric and oceanic mass variations. The GRACE products may be validated against in situ OBP observations which are available from a ground truth site in the tropical northwest Atlantic Ocean. Large differences are observed between the in situ and GRACE-derived OBP which are investigated by comparing the tidal and nontidal ocean models used at GFZ and UTCSR for dealiasing short-term ( $<2$ months) mass variations from satellite measurements. Results show that the barotropic nontidal and tide models need improvement at periods shorter than 1 day and longer than 2 weeks. On a global scale the monthly OBP fields from GRACE generally overestimate the variability compared to ocean general circulation models, especially in tropical regions. This may be attributed to continuing deficiencies in GRACE data processing. Nevertheless, there is some initial evidence that GRACE possesses the potential to observe large-scale averages of bottom pressure fluctuations.

Citation: Kanzow, T., F. Flechtner, A. Chave, R. Schmidt, P. Schwintzer, and U. Send (2005), Seasonal variation of ocean bottom pressure derived from Gravity Recovery and Climate Experiment (GRACE): Local validation and global patterns, J. Geophys. Res., 110, C09001, doi:10.1029/2004JC002772.

\section{Introduction}

[2] One of the key goals of the Gravity Recovery and Climate Experiment (GRACE) [Tapley and Reigber, 2004] is measurement of the variability of ocean bottom pressure (OBP) from monthly maps of Earth's gravity field. Assuming that monthly gravity changes over oceanic regions are caused entirely by mass redistribution above the ocean bottom (i.e., within the ocean and atmosphere), they can be transformed to fluctuations in OBP at

\footnotetext{
${ }^{1}$ Leibniz-Institut für Meereswissenschaften an der Universität Kiel (IFM-GEOMAR), Kiel, Germany.

${ }^{2}$ Department 1 (Geodesy and Remote Sensing), GeoForschungsZentrum Potsdam (GFZ), Potsdam, Germany. USA.

${ }^{3}$ Woods Hole Oceanographic Institution, Woods Hole, Massachusetts,

${ }^{4}$ Deceased 24 December 2004.
}

Copyright 2005 by the American Geophysical Union. 0148-0227/05/2004JC002772\$09.00 colatitude $\varphi$ and longitude $\lambda$ as described by Wahr et al. [2002]:

$$
\begin{aligned}
\Delta \operatorname{OBP}(\varphi, \lambda)= & \frac{a g \rho_{E}}{3} \sum_{l=0}^{\infty} \sum_{m=0}^{l} \frac{(2 l+1)}{\left(1+k_{l}\right)} \tilde{P}_{l m} \cos (\varphi) \\
& \cdot\left[\Delta C_{l m} \cos (m \lambda)+\Delta S_{l m} \sin (m \lambda)\right]
\end{aligned}
$$

where $a$ is the Earth's semimajor axis, $g$ is the mean gravitational acceleration, $\rho_{E}$ is the mean density of Earth, $P_{l m}$ are normalized Legendre polynomials of degree $l$ and order $m$, the $k_{l}$ are Love numbers parameterizing the response of the solid Earth to surface loads, and $\Delta C_{l m}$ and $\Delta S_{l m}$ are the normalized spherical harmonic coefficient differences of two gravity field solutions separated in time.

[3] In situ OBP measurements provide a tool to study the abyssal flow field using the geostrophic relationship [e.g., Luther and Chave, 1993]. At points distant from oceanic boundaries and the equator, on subinertial timescales, and for spatial scales large compared to the water depth, 
horizontal motions are to the lowest-order approximation geostrophic, so that:

$$
\vec{v}_{h}=\frac{1}{f \rho} \vec{e}_{3} \times \nabla_{h} p
$$

where $\vec{\nu}_{h}, f, \rho, \vec{e}_{3}$ and $p$ denote the horizontal water velocity, Coriolis parameter, water density, a unit vector in the zenith direction, and in situ pressure, respectively. Pedlosky [1987] shows that a simple relationship between the pressure difference at two points in the horizontal plane and the mass flux per unit distance obtains from (2)

$$
p(\xi)-p\left(\xi_{o}\right)=f \int_{\xi_{o}}^{\xi} \vec{e}_{3} \bullet\left(\rho \vec{v}_{h} \times d \vec{r}\right)
$$

where $p(\xi)>p\left(\xi_{o}\right)$ and $d r$ is an incremental vector parallel to an arbitrary curve running from $\xi_{o}$ to $\xi$.

[4] The existing in situ OBP database is extremely sparse in time and space. However, if the OBP spatial differences could be inferred from GRACE with an accuracy of 0.1 mbar over scales of order $500 \mathrm{~km}$, global monitoring of the deep flow field would be feasible [Hughes et al., 2000].

[5] In order to provide accurate monthly gravity fields, or equivalently via equation (1), OBP maps, careful dealiasing of the monthly GRACE gravity field solutions for shorterterm mass variations is performed in the two processing centers at GeoForschungsZentrum Potsdam (GFZ) and the University of Texas Center for Space Research (UTCSR). In addition to atmospheric mass variations, the effect of ocean tides and short-period (below the two month Nyquist period) barotropic variability have to be accounted for. Wunsch [1997] notes that up to $50 \%$ of the horizontal eddy kinetic energy in the ocean is barotropic, and barotropic timescales are typically short compared to baroclinic ones, so that barotropic fluctuations are comparatively large at periods under two months. The GFZ and UTCSR gravity field products are based on the same instrumental data, and the processing methods are similar. Both centers apply a dynamic orbit adjustment which includes estimation of unknowns such as initial state, numerous instrument parameters, and gravity field spherical harmonic coefficients. The orbital arc length (orbit integration length) is 1.5 days at GFZ and 1 day at UTCSR. A nominal arc length of 1.5 days was selected at GFZ as a compromise between the need for short arcs to prevent an increase of residual errors in nongravitational accelerations, and long arcs to cover at least one half of GRACE's primary resonance period. GPS data, necessary for precise positioning of the GRACE satellites, are analyzed as original code and phase measurements at GFZ and double differenced at UTCSR. Short temporal-scale oceanic motions are corrected using identical (barotropic nontidal ocean model, planetary ephemerides) or similar (atmospheric tides) correction models. One important difference lies in the ocean tide models: the FES2002 model [LeProvost, 2002] used at GFZ is based on a combined hydrodynamic and assimilation approach, while the CSR4.0 model (CSR4.0 Global Ocean Tide Model, available at ftp://ftp.csr.utexas.edu/pub/tide/) used at UTCSR is empirical.
[6] The goal of this paper is to present an initial evaluation of the GRACE time-varying gravity field over oceanic regions through comparison with in situ OBP measurements. Previously, GRACE accuracy has been inferred only from simulated mission data [Condi and Wunsch, 2004]. Section 2 gives a brief description of the dealiasing procedure and the tidal models used in GRACE data processing. Overviews of the GRACE products used in this study and of data from the ground truth site are given in sections 3 and 4, respectively. The GRACE-derived OBP and in situ ground truth measurements are then compared in section 5. In addition, the output from the barotropic ocean model used for nontidal dealiasing and the GFZ and UTCSR ocean tide models are validated against in situ measurements from the ground truth site to identify possible modeling errors. In section 6, the global GFZ and UTCSR GRACE-derived OBP fields are contrasted. Further OBP comparisons are presented based on averages over the North Atlantic derived from GRACE and from an ocean circulation model constrained by observations [Stammer et al., 2003]. The results provide encouraging evidence that at this early stage of the experiment, GRACE might have some capacity in recovering a large-scale annual OBP cycle.

\section{Reduction of Time-Variable Gravity in GRACE Products}

[7] In order to derive small OBP fluctuations from monthly gravity field solutions, the GRACE observations must be corrected for time-varying gravitational phenomena at periods shorter than the Nyquist value (i.e., periods shorter than 2 months), including Earth and ocean tides and other atmospheric and oceanic mass variations. This is done with prognostic models during the reduction of the GRACE satellite-to-satellite tracking data at both level 2 processing centers. In the context of understanding GRACE-derived OBP variations, the models for the nontidal atmospheric and oceanic mass variations and the ocean tides are of particular interest and shall be briefly described. All other aspects of GRACE data reduction are described in detail in the GFZ and UTCSR level 2 processing standard documents [Flechtner, 2003a; Bettadpur, 2004]. A description of the dynamic approach for GRACE gravity field determination at GFZ is also given by Reigber et al. [2005].

[8] For removing the influence of ocean tides, GFZ has adopted the FES2002 model [LeProvost, 2002], whereas UTCSR uses the CSR4.0 model (CSR4.0 Global Ocean Tide Model, available at ftp://ftp.csr.utexas.edu/pub/tide/). Both models include the same principal tides in the longdiurnal, diurnal, and semidiurnal period bands. However, since the models are derived from different data using distinct approaches, inevitable discrepancies will impact the OBP estimates derived from the GFZ and UTCSR gravity field models in different ways, as is further discussed in section 6 . A related topic discussed in section 5 is the shortcomings of current state-of-the-art global ocean tide models in the semidiurnal band [Knudsen, 2003].

[9] Nontidal atmospheric and ocean mass variations are accounted for using standard GRACE atmosphere and ocean dealiasing products (AOD) [Flechtner, 2003b], which consist of sets of spherical harmonic corrections terms complete up to degree and order 100 at 6 hour intervals 
$(0,6,12$, and 18 hours UTC) that are applied to the static gravity field during dynamic orbit determination. For the atmospheric part, the input data for the expansions are global $0.5^{\circ} \times 0.5^{\circ}$ multilayer grids of meteorological fields from the European Center for Medium-Range Weather Forecasts (ECMWF), which are integrated vertically to yield the 3D atmospheric mass distribution at each 6 hour epoch. The nontidal oceanic mass variations are derived from a barotropic ocean model, which is hereafter denoted PPHA because it was originally developed by Pacanowski, Ponte, Hirose and Ali [Ponte, 1993; Hirose et al., 2001]. The PPHA model is forced by 6-hourly ECMWF meteorological fields and computes the component of oceanic mass redistribution ("barotropic sea level") due to wind stress and atmospheric pressure for an area between $65^{\circ} \mathrm{N}$ and $75^{\circ} \mathrm{S}$. It lacks baroclinic dynamics and surface buoyancy forcing which are commonly believed to have little effect at timescales below one month [Philander, 1978], but the deficiencies may be critical for the timescale from one to two months, as is further discussed in section 5 .

\section{GRACE Products and Analysis Procedure 3.1. Monthly Gravity Field Solutions}

[10] The detided and dealiased GFZ and UTCSR monthly averaged GRACE GSM (GRACE Satellite-only Model) gravity field solutions used in this study are listed in Table 1.

\subsection{Nontidal Monthly Averaged Dealiasing Products}

[11] As described in section 2, short-term nontidal variations in the atmosphere and oceans were removed from the GRACE data using the 6-hourly AOD products. As a result, differences between monthly GSM solutions should reflect mainly continental hydrological phenomena [Schmidt et al., 2005] and long-period barotropic fluctuations of the ocean if instrument measurement noise and mismodeling of shortterm mass variations are negligible. If the monthly averaged mass variations are added back into the dealiased GSM products, monthly OBP fluctuations can be studied on a global scale using equation (1). Therefore so-called GRACE Average of Nontidal Atmosphere and Ocean Combination (GAC) products are provided by the processing centers, which are monthly averages of AOD products used to generate each monthly GSM field noted in Table 1.

[12] Errors in the higher-order terms of the spherical harmonic series, unresolved deficiencies in data processing (such as mismodeled or unmodeled short-term mass variations), and variable spatial sampling due to the ground track evolution from month to month can add spurious short wavelength meridional oscillation patterns to the monthly fields [Wiehl and Dietrich, 2005]. These are easily detected by plotting the differences between any two monthly solutions, and resemble aliasing errors as simulated by Han et al. [2004]. To minimize this problem, a spatial domain Gaussian filter with a $1000 \mathrm{~km}$ half width (corresponding approximately to degree and order 20) was applied to the full GSM and GAC products [Wahr et al., 2002], which were then truncated at degree and order 50, corresponding to a spatial resolution of $400 \mathrm{~km}$. The $\mathrm{C}_{20}$ (flattening) coefficient has been excluded from the expansion (as done in earlier studies [see Wahr et al., 2004; Tapley et al., 2004]) because it still shows unrealistic variability
Table 1. Monthly Averaged GRACE Gravity Field Products (GSM) Available From GFZ and UTCSR Processing Centers ${ }^{\mathrm{a}}$

\begin{tabular}{lccc}
\hline Month & Year & GFZ & UTCSR \\
\hline April/May & 2003 & X & X \\
August & 2002 & X & X \\
November & 2002 & X & X \\
February & 2002 & - & X \\
March & 2003 & X & X \\
April & 2003 & X & X \\
May & 2003 & $X$ & X \\
July & 2003 & $X$ & X \\
August & 2003 & $X$ & X \\
September & 2003 & - & X \\
October & 2003 & $X$ & - \\
November & 2003 & $X$ &
\end{tabular}

${ }^{\mathrm{a}}$ Direct comparisons between GFZ and UTCSR fields can be carried out with 9 of the 12 monthly solutions.

between the monthly solutions, which is still not completely understood. A possible reason could be the high correlation between $\mathrm{C}_{20}$ and the $\mathrm{K}$ band parameterization.

\subsection{Ancillary Data and Model Output}

[13] PPHA OBP, as well as ECMWF sea level pressure (SLP), is available at 6 -hour intervals on a $0.5^{\circ}$ grid for the time period January 2002 through December 2003. FES2002 and CSR4.0 ocean tide amplitudes and phases were transformed into spherical harmonic expansions of degree and order 50 representing the tide-induced OBP with a temporal resolution of one hour over April to December 2002.

\section{OBP Ground Truth Site}

[14] In this study, OBP data from the Meridional Overturning Variability Experiment (MOVE) located in the tropical northwest Atlantic were used for GRACE validation. MOVE is designed to measure fluctuations in the meridional mass transport of the deep ocean by means of OBP and density monitoring [Kanzow, 2000, 2004; Send et al., 2002; T. Kanzow et al., Monitoring the integrated deep meridional flow in the tropical North Atlantic: Long-term performance of a geostrophic array, submitted to Deep-Sea Research, 2005, hereinafter referred to as Kanzow et al., submitted reference, 2005].

[15] Since January 2000, OBP time series based on Paroscientific quartz pressure transducers have been acquired at the mooring sites M1, M2 and M3 (see Figure 1). In February 2004, four additional OBP sites (M4-M7) were added to ensure enhanced spatial coverage of the OBP signal. The February 2004 instruments will be recovered in 2006, and were not available for this study.

[16] The zonal M1-M3 axis spans a distance of about $1000 \mathrm{~km}$. As a result, GRACE versus MOVE comparisons of the seasonal evolution of OBP at three single sites can be carried out, and the zonal OBP gradient (e.g., the M1-M3 difference of OBP fluctuations) can also be examined. The latter yields the meridional near-bottom geostrophic velocity fluctuations according to (2). Thus aspects of the dynamics of the abyssal flow as observed by GRACE can also be assessed.

[17] The OBP recorders were recovered and redeployed at approximately annual intervals. Long-term instrument 


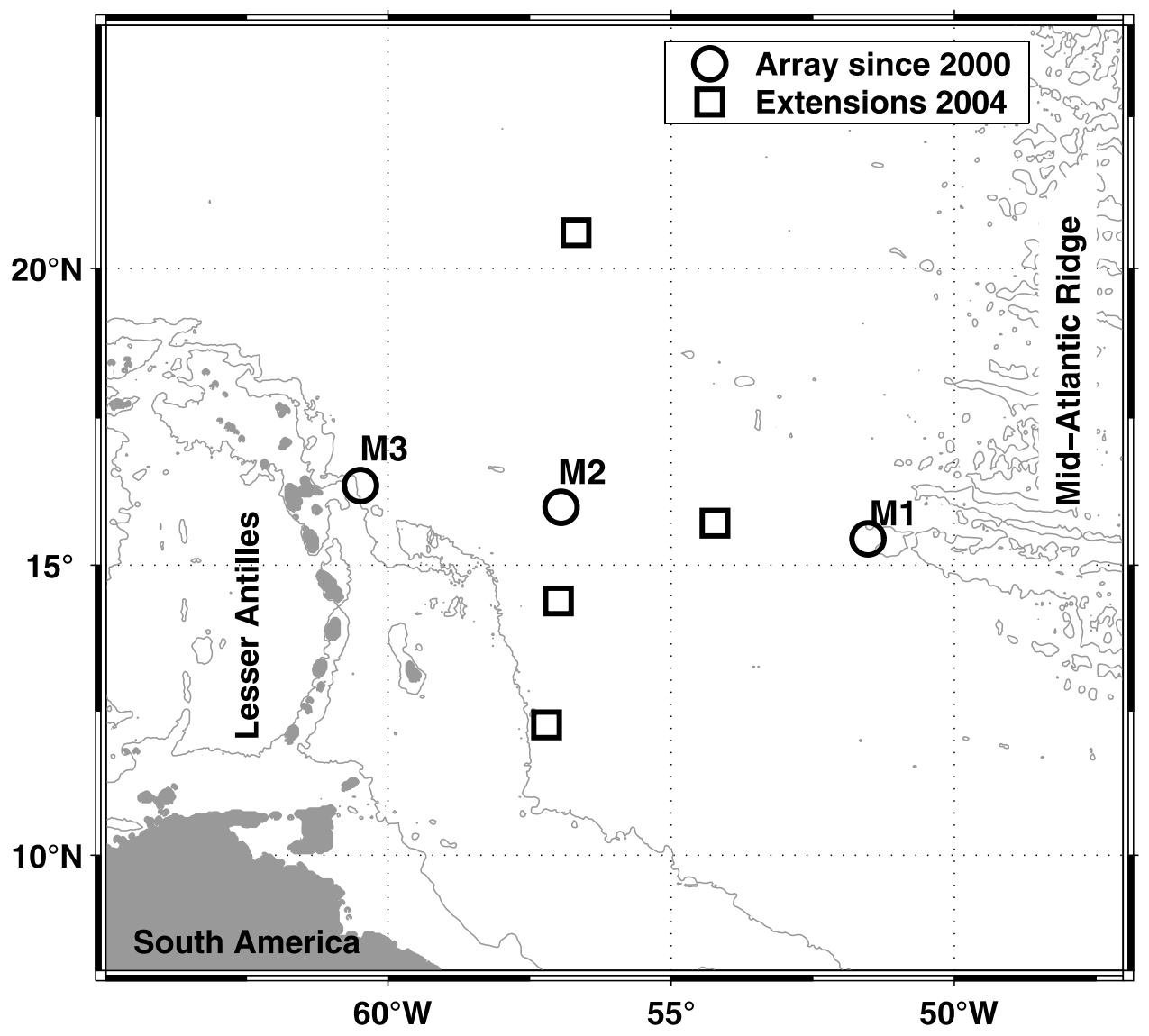

Figure 1. The MOVE ground truth site in the tropical northwest Atlantic, bounded by the Lesser Antilles arc to the west and the Mid-Atlantic Ridge to the east. At three sites along the zonal line (M1, M2, and M3), time series of OBP have been acquired since January 2000.

drift was subtracted from each segment as described by Kanzow et al. (submitted manuscript, 2005). For comparison with OBP inferred from both GRACE and nontidal barotropic models, the tides have to be eliminated from the data. Unless explicitly stated otherwise, diurnal and semidiurnal tides have been removed using a 48-h 6 pole Butterworth low-pass filter. Longer-period tides (fortnightly and monthly) were subtracted explicitly using harmonic analysis [Lohmann, 1999].

\section{Validation of GRACE OBP Using in Situ Measurements}

[18] Comparison between GRACE and in situ measurements should be carried out in regions where the horizontal correlation scale of the true OBP signal is larger than the $1000 \mathrm{~km}$ scale of the spatial filter applied to the satellite OBP. This is generally the case on the MOVE line: the OBP at sites M1 and M3 (separated by roughly $1000 \mathrm{~km}$ ) have a magnitude squared coherence $>0.6$ over the band from a few days to several months. Peak values approaching 1.0 are observed at periods below 8 days.

[19] A comparison between MOVE and GRACE OBP immediately shows that GRACE strongly overestimates the variability (Figure 2, top). While GRACE OBP amplitudes peak at 5 mbar, the in situ observations barely reach 1 mbar. Further, the GRACE OBP signal is only weakly correlated with the in situ measurements. However, there is a high correlation among the GRACE time series at M1 and M3, as there is among the in situ observations. The fluctuations of the zonal M1-M3 OBP gradient (Figure 2, bottom), which correspond to meridional velocity fluctuations (see scale at the right-hand side of Figure 2), are also overestimated by GRACE, although not by the same amount as for OBP. There appears to be more similarity to the MOVE OBP gradient (at least in amplitude) during 2003. This occurrence corresponds with GRACE satellite software upgrades which have led to continuously improving data quality since early 2003, and suggests that GRACE has a marginal capability to observe spatial OBP gradients using monthly solutions. Nevertheless, the differences between the GRACE and MOVE OBP gradients remain large.

[20] The capability of GRACE to recover OBP gradients might be dependent on the spatial scale. The exclusion of the flattening term $\mathrm{C}_{20}$ from the GSM and GAC spherical harmonic expansions only affects the largest wavelengths $(20,000 \mathrm{~km})$. Its inclusion would have caused even larger inferred seasonal fluctuations, but would not affect the spatial gradient on scales of a few thousand kilometers. If the GRACE OBP fields are contaminated by erroneous long wavelength noise, this could explain why the temporal evolution of the GRACE OBP M1-M3 gradient compares better to in situ observations than the OBP time series at M1 and M3. However, note that the $1000 \mathrm{~km}$ spatial filter 

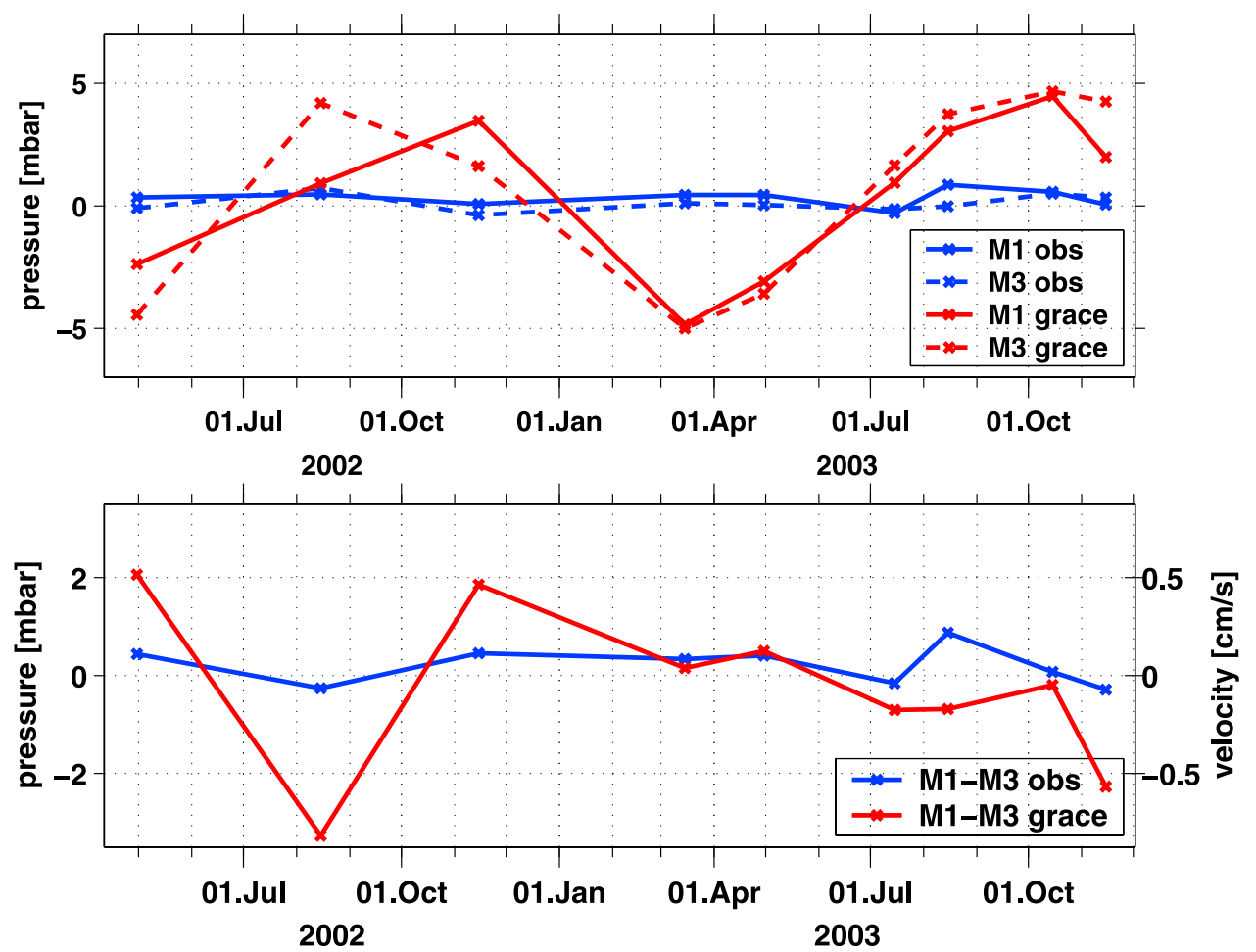

Figure 2. Comparison of (top) GRACE (red) and in situ (blue) OBP fluctuations and (bottom) horizontal M1-M3 differences of OBP fluctuations.

applied to the GRACE data corresponds approximately to the separation between M1 and M3. As a consequence, the GRACE time series at the two sites are not independent. This may partly explain the high degree of correlation between GRACE OBP at M1 and M3, which will also reduce the magnitude of the GRACE M1-M3 OBP gradient.

[21] Initial results indicate that GRACE realistically resolves the strong annual continental hydrological cycle [Schmidt et al., 2005; Tapley et al., 2004; Wahr et al., 2004] with an amplitude of about 10 mbar. A key question which must be answered is what could cause the large root mean square (RMS) oceanic difference of 3.4 mbar between OBP fluctuations from in situ observations and GRACE at the MOVE site. This discrepancy agrees well with the $3.5 \mathrm{mbar}$ uncertainty of GRACE to recover continental hydrological signals when using a $750 \mathrm{~km}$ filter averaging radius [Schmidt et al., 2005].

[22] Errors in the MOVE OBP measurements amount to only about 0.2 mbar on timescales shorter than 1 year (Kanzow et al., submitted manuscript, 2005). Numerical simulations indicate that the expected amplitude of the annual OBP cycle over the oceans typically is about an order of magnitude lower than the hydrological signal over most continental regions [e.g., Condi and Wunsch, 2004]. This makes the oceanic annual cycle much harder to detect. Additionally, the dealiasing of GRACE data over the oceans is far more complicated than that over land. The amplitudes of the fast moving tidal and nontidal barotropic fluctuations which have to be accurately eliminated are much larger than those of the monthly OBP fluctuations that ought to be recovered by GRACE. Thus the accuracy of dealiasing the
GRACE observations depends crucially on the capability of numerical models to predict true ocean variability. In the sequel, this will be assessed by comparing the nontidal and tidal models used for GRACE data reduction with observations from the MOVE site. Note that short-term terrestrial hydrological variability will also contribute to aliasing errors [Han et al., 2004], especially near large river outflows.

[23] The barotropic PPHA model OBP compares well with the amplitude and phase of the in situ observations at sites M1 and M3 (Figure 3). Magnitude squared coherence of 0.70 (where the zero coherence level at $95 \%$ significance is 0.30 ) between modeled and observed OBP is observed at periods below 20 days at both sites. Both data sets are dominated by high-frequency variability (Figures 3, top and 3 , middle). The RMS amplitudes of the MOVE observations and the PPHA model amount to 1.65 and $1.22 \mathrm{mbar}$, respectively. On the other hand, the temporal fluctuations of the M1-M3 OBP gradient are significantly underestimated by PPHA, as the model RMS amplitude of 0.29 mbar is only one third of the observed value of 0.91 mbar. The ocean dynamics in the model are obviously too weak. Small but statistically significant magnitude squared coherence ( 0.38 on average, where the zero coherence level at $95 \%$ significance is 0.30 ) between the modeled and measured pressure gradient is observed in the 6 day to 3 month band. It is reasonable to assume that aliasing effects will be strongest in the presence of short-period but long wavelength fluctuations. The discrepancy between model and observation is significantly larger for the M1-M3 OBP difference than for the M1 or M3 OBP. This could be explained by the presence of a signal with a wavelength 


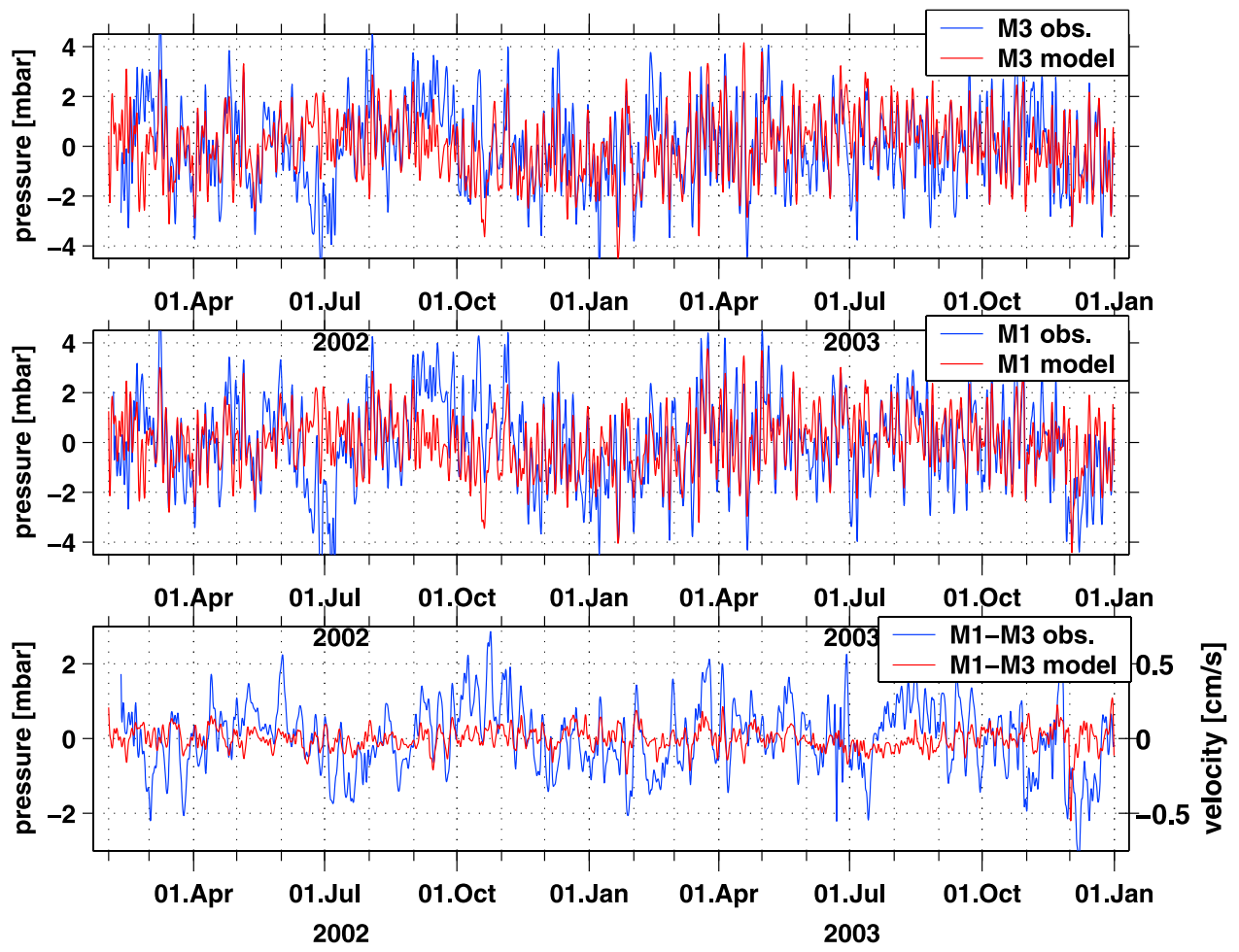

Figure 3. OBP derived from in situ observations (blue) and from the barotropic PPHA model (red) at sites (top) M3 and (center) M1. (bottom) M3-M1 OBP differences. Note the corresponding velocity scale on the right. All time series are 2 day low-pass filtered. Additionally, the monthly and fortnightly tides in the observations were eliminated using the harmonic fit of Lohmann [1999].

much shorter than the M1-M3 separation of $1000 \mathrm{~km}$ in the model, which would not result in severe aliasing effects. However, analyses of the in situ observations suggest that much of the variability seen in the M1-M3 difference originates from barotropic Rossby waves whose wavelengths exceed $1000 \mathrm{~km}$ in the 6-15 day band [Kanzow, 2004].

[24] To assess possible model deficiencies, a spectral analysis of modeled and observed OBP and OBP gradients has been carried out (Figure 4). The PPHA model reaches the observed energy levels in period bands only between 2 and 6 and near 15 days. The modeled variability is significantly underestimated at longer periods extending up to 60 days, which may lead to significant errors in the monthly gravity field solutions. Further, the modeled OBP gradient strongly underestimates the observed gradient at all frequencies. This obvious mismodeling at lower frequencies will surely affect the quality of the dealiasing and therefore contaminate the monthly GRACE solutions.

[25] The obvious model limitations at periods longer than a few weeks are a critical issue and may be attributed to the absence of nonatmospherically forced barotropic dynamics such as radiation from jets [e.g., Kamenkovich and Pedlosky, 1998a, 1998b], the lack of baroclinic dynamics, errors in parameterizing barotropic dissipation, and other simulation problems. For example, Miller et al. [1987] demonstrated that a boundary current forced by only mean winds can readily fill a flat-bottomed numerical model basin with barotropic energy radiated from the meandering cur- rent after it separates from the boundary. Song and Zlotnicki [2004] showed that baroclinic features like tropical instability waves may cause strong OBP fluctuations at periods around 30 days, which could alias into the GRACE monthly solutions. Replacement of the PPHA barotropic model with a full ocean general circulation model might be expected to lead to enhancement of the GRACE monthly gravity field solutions over the oceans. However, barotropic fluctuations in the oceans are very poorly characterized relative to baroclinic ones because most measurements are insensitive to its presence. The barotropic component is invisible to hydrography, difficult to separate from the baroclinic field in mooring data [e.g., Wunsch, 1997], and even sea surface heights from satellite altimeters is usually dominated by baroclinic variability at subinertial periods as a consequence of the vertical mode structures of barotropic and baroclinic components, in combination with the slightly higher energy level of baroclinic variability. As a result, the barotropic data constraints on any numerical model are weak, and hence errors in any barotropic simulation are expected to be commensurate.

[26] At present, only timescales ranging from a few days to about one year have been analyzed using GRACE. Because the AOD dealiasing coefficients are available with 6 hour resolution and are interpolated in space and time during satellite orbit integration for gravity field recovery, the PPHA performance on very short timescales is of particular interest. In Figure 5 a 30 day model data segment near site M1 is compared with in situ observations. For this comparison, the tidal constituents in the 


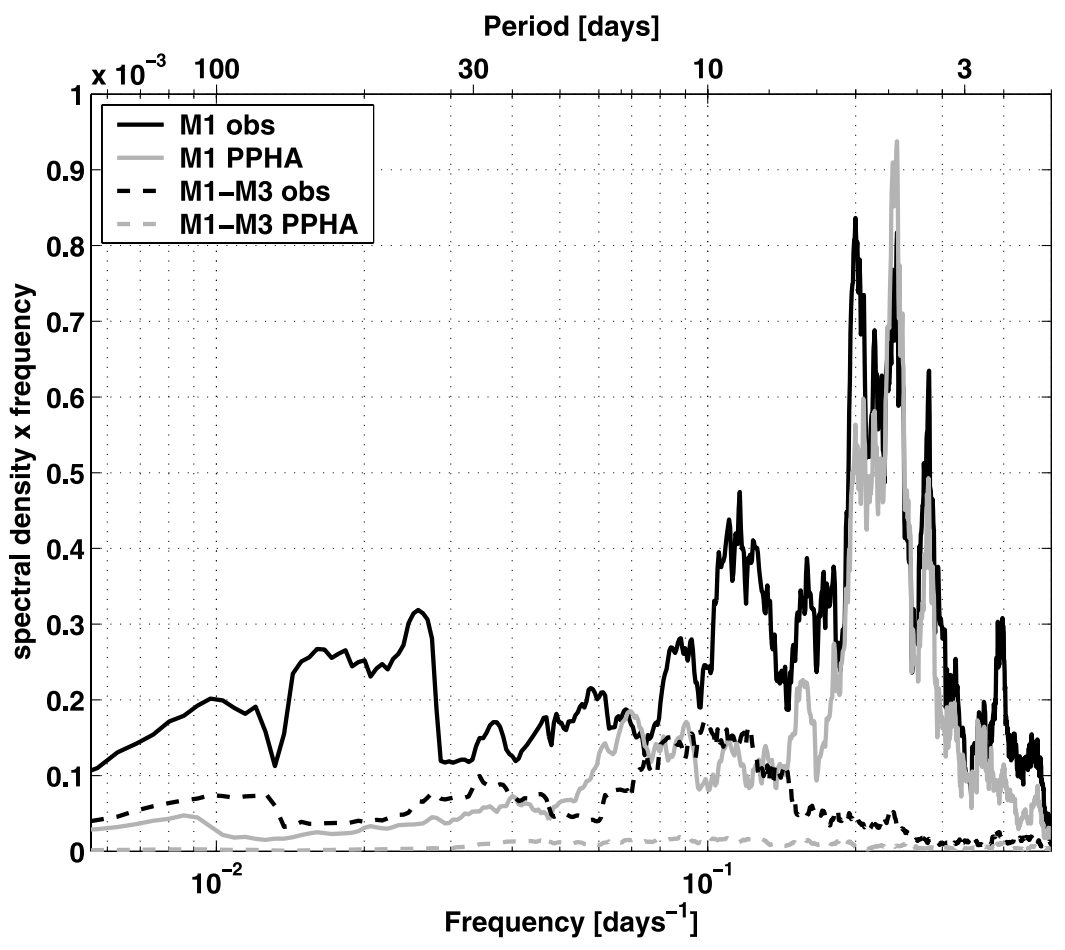

Figure 4. Variance conserving spectra of the time series from Figure 3. Spectra were estimated using the multitaper method with a time bandwidth of 5 and have a bandwidth of $0.0072 \mathrm{cpd}$ and approximately 18 equivalent degrees of freedom at each frequency.

half-day to monthly band have been removed from the in situ observations using a harmonic fit [Lohmann, 1999].

[27] The ECMWF SLP used to force the PPHA model displays a 6-hour sawtooth-like characteristic (Figure 5). Moreover the modeled OBP is found to vary in phase with the SLP forcing on this timescale with the former exhibiting much larger amplitudes than the latter. These fluctuations could be explained by the presence of atmospheric tides which have not been eliminated in the PPHA SLP forcing, and which are not present in the in situ OBP time series since the tides have been removed. To allow for an objective comparison, the high-frequency atmospheric contribution (i.e., the thin solid line in Figure 5) has been added to the in situ observations. Even then, Figure 5 suggests that the PPHA model overestimates the variability on 6 hour timescales. The RMS amplitude of the 6-hour OBP difference is 1.9 mbar for the observed (including the short-term SLP signal) and 3.2 mbar for the modeled data. Overestimation of variability on these short timescales will contribute to aliasing effects in the GRACE gravity field since the 6-hour fluctuations will be folded to a 12 hour period. As has been pointed out by Knudsen [2003], due to the sampling characteristics of GRACE's orbit, the half-day tidal constituent S2 is associated with an alias frequency of 162 days. Similar arguments would apply to the high-frequency fluctuations in the PPHA model.

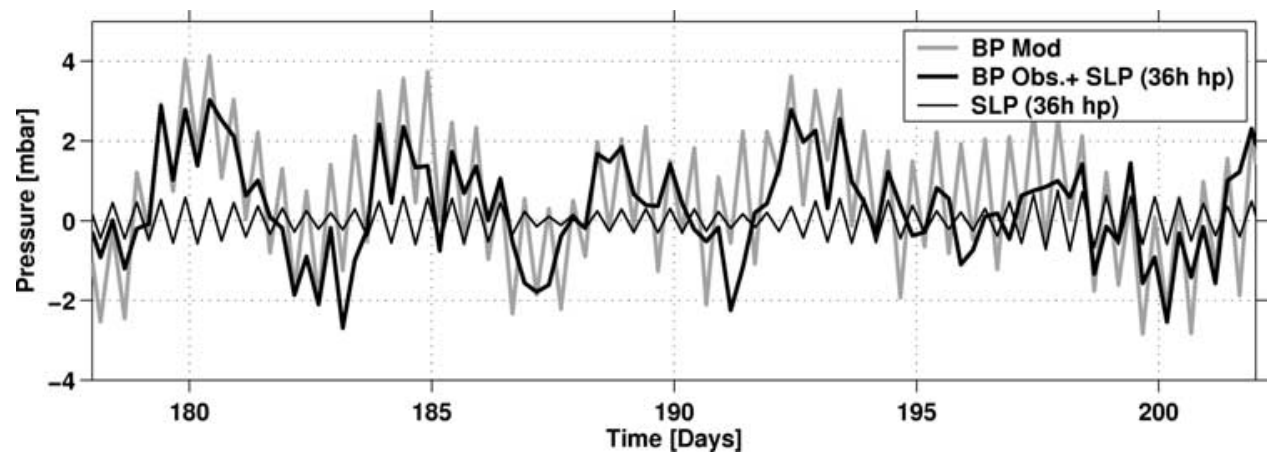

Figure 5. High-frequency OBP fluctuations from the PPHA model near site M1 (shaded line) and the M1 in situ observations (thick solid line). A set of 48 semidiurnal, diurnal, fortnightly, and monthly tides were removed using a harmonic fit [Lohmann, 1999]. The 36 hour high-pass-filtered SLP used to force the PPHA model (thin solid line) has been added (see text for details). 

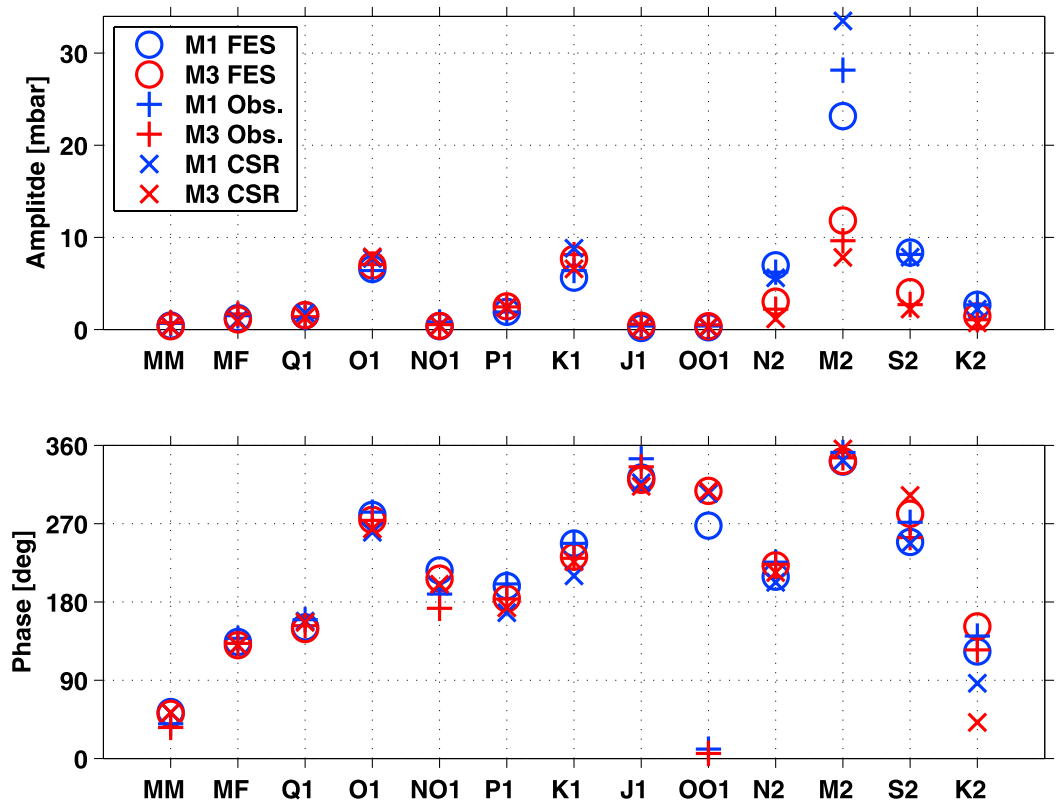

Figure 6. Comparison of tidal constituents from in situ observations as well as from the FES2002 and CSR4.0 ocean tide models. (top) Amplitudes and (bottom) phases of the 13 largest tides obtained by applying a harmonic fit to the respective time series [Lohmann, 1999]. The phase is indicated relative to the beginning of the time series.

[28] At these short timescales, a full ocean general circulation model would be unlikely to lead to major improvements, as most barotropic and baroclinic signals are confined to longer timescales. A better representation of the atmospheric tides with increased temporal resolution [Ponte and Ray, 2002] in the meteorological forcing fields could possibly reduce the noise level. Such a product is not currently available. Han et al. [2004] have shown that mismodeling of the atmosphere may lead to significant aliasing effects in the GRACE gravity solutions that exceed measurement noise. The ensuing errors in the monthly solutions are manifest as pervasive narrow meridional stripes.

[29] A further candidate explanation for overestimation of OBP fluctuations by GRACE is errors in the oceanic tide dealiasing [Knudsen, 2003; Han et al., 2004]. To investigate this, nine month time series of hourly OBP data (see section 3) derived from the FES2002 and CSR4.0 ocean tide models were compared to the observed OBP data at the MOVE site. A harmonic fit that includes 48 tidal constituents in the band covering the semidiurnal to monthly components was applied to the tide model output as well as to the in situ OBP time series using the algorithm developed by Lohmann [1999]. In Figure 6 the amplitude (Figure 6, top) and phase (Figure 6, bottom) of the largest tidal constituents are displayed. Generally, there is very good agreement between the modeled and observed amplitudes and phases except for the semidiurnal M2 and S2 tides. The M2 tide amplitude in observed OBP reaches about 28 mbar at site M1 and $10 \mathrm{mbar}$ at site M3. At site M1, the difference between the CSR4.0 and FES2002 M2 amplitudes is 10 mbar, with CSR4.0 overestimating and FES2002 underestimating the observed values by about 5 mbar, respectively. At site M3 the situation is reversed; CSR4.0 underestimates and FES2002 overestimates the observed M2 value but by only 2 mbar. Because the harmonic fit introduces errors of less than 1 mbar, the substantial difference of 10 mbar in the estimate of the M2 tide can be regarded as significant. By contrast, the model phases of all of the major tidal constituents compare well with the observed OBP.

[30] To quantify the impact of tide model errors on the monthly GRACE gravity fields, we refer to the findings of Knudsen [2003]. He points out that different tide constituents are associated with different alias frequencies according to the GRACE orbit sampling characteristics. The dominant M2 tide is associated with an alias period of 13.6 days, leading to a reduction in the tide errors of $90 \%$ for the monthly averages. A $90 \%$ reduction of a 5 mbar error (i.e., half the difference between the estimates of FES2002 and CSR4.0) in mismodeling M2 at mooring site 1 yields a residual monthly error of 0.5 mbar. Spatial aliasing patterns in the monthly GRACE solutions associated with M2 tide misrepresentation comprise meridional bands of alternating negative and positive values [Han et al., 2004]. These can be drastically reduced by applying a Gaussian filter with a horizontal radius of $800 \mathrm{~km}$. However, S2 and K2 exhibit alias periods of 162 and 1460 days, respectively. Therefore mismodeling of those tide constituents is reduced by only $6 \%$ and $0 \%$, respectively, when monthly averages are applied [Knudsen, 2003]. Aliasing of S2 appears to be associated with a long-wavelength pattern, which cannot be smoothed with a reasonable spatial filter [Han et al., 2004]. Mismodeling of S2 and K2 at the MOVE site does not exceed 1 mbar and 0.3 mbar, respectively (see Figure 6), which might nevertheless affect the GRACE OBP significantly since the expected annual OBP cycle has a comparable amplitude [e.g., Condi and Wunsch, 2004]. Errors in 

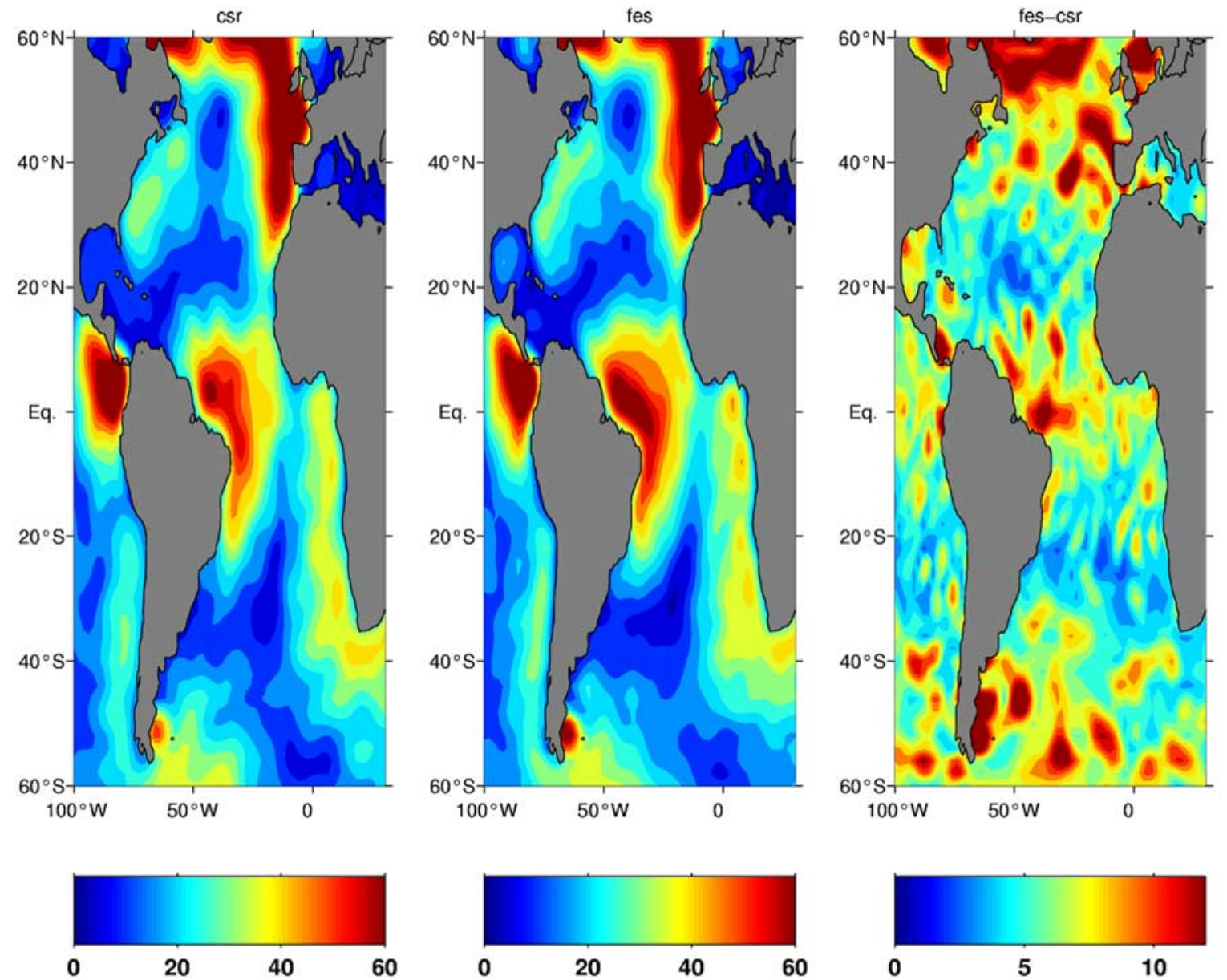

Figure 7. RMS amplitude in mbar from (left) the CSR4.0 and (middle) the FES2002 ocean tide models. (right) RMS amplitude of the difference between the two models. Computations are based on hourly model output between April and December 2002.

all diurnal tide constituents average out almost completely in the monthly fields [Knudsen, 2003] because their alias periods are about one day.

[31] On the basis of these results, further investigations should focus on tide dealiasing as well as subinertial barotropic model improvement. However, apart from S2, which has the potential to introduce a long-period, long wavelength aliasing pattern, other tide constituents with shorter alias periods can be reduced by applying suitable spatial filters to the GRACE data. Figure 7 extends this analysis to the whole Atlantic. Visually, the RMS amplitudes of the 9 month time series (see section 3) of CSR4.0 and FES2002 (left and center panel, respectively) agree well with each other. Both models display maximum amplitudes of more than $60 \mathrm{mbar}$ in the tropical West Atlantic and in the northeast Atlantic, whereas in the subtropical ocean, much smaller differences are seen. However, the RMS amplitude of the intermodel difference can reach 15 mbar or more in some locations. The true errors of the tide models could be even larger than their relative differences since they are based in part on the same data. It is not surprising to observe large differences in regions where the tide amplitudes themselves are large. Nevertheless, there are locations in the South Atlantic between $40^{\circ}$ and $60^{\circ} \mathrm{S}$, as well as in the northern North Atlantic, where the tidal amplitudes are comparatively small, but where the CSR4.0 and FES2002 RMS differences reach 15 mbar. A closer analysis reveals that the tide phases differ substantially between the models in those regions.

\section{GRACE Large-Scale OBP Patterns}

[32] In ocean models, the flow patterns on timescales longer than a few months are typically zonally extended [e.g., Condi and Wunsch, 2004]. However, such patterns are not observed in the GRACE OBP solutions.

[33] Figure 8 shows the OBP standard deviation computed from the nine monthly OBP solutions (see Table 1) from UTCSR (upper panel) and GFZ (middle panel). OBP from both processing centers displays the same level of overall variability, but differs in detail. The most prominent feature in both cases is leakage of the tropical continental hydrological cycle into the ocean at its lateral boundaries between $20^{\circ} \mathrm{N}$ and $20^{\circ} \mathrm{S}$, which is due to the coarse resolution of the applied spatial filter. Zonally coherent patterns cannot be discerned in either product. Instead, GRACE OBP seems to be dominated by meridionally coherent patterns. 

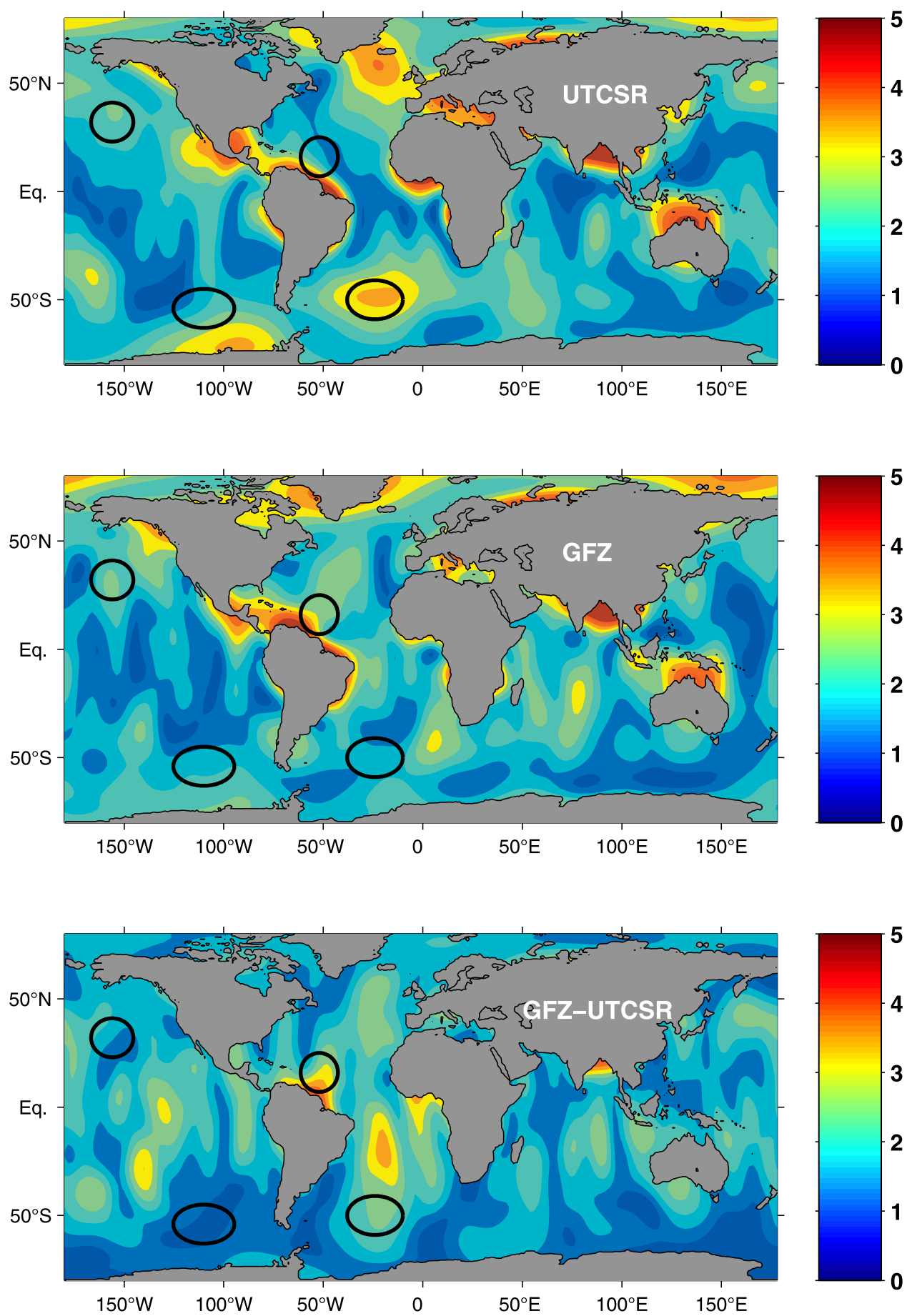

Figure 8. Standard deviation in mbar of OBP inferred from the (top) UTCSR and (middle) GFZ monthly GRACE gravity field solutions. (bottom) Standard deviation of the UTCSR and GFZ OBP difference.

Additionally, ocean models on these timescales display a typical OBP variability of about 1 mbar [e.g., Condi and Wunsch, 2004], which is only about half of that seen in the GRACE-derived OBP.

[34] In the differences between the UTCSR and GFZ OBP (Figure 8, bottom), the continental hydrological leakage signal vanishes almost completely. This means that its predicted amplitude as well as its predicted phase agrees well between the two GRACE products. Instead, the pattern of differences emphasizes meridionally oriented characteristics. On the basis of the prior discussion, we interpret this as an aliasing pattern introduced by tidal and nontidal mismodeling as well as instrument measurement noise. No clear relationship between this pattern and the differences between the FES2002 and CSR4.0 ocean tide models (Figure 7, right) can be discerned. The best agreement between GFZ and UTCSR OBP is seen at high latitudes (north of $60^{\circ} \mathrm{N}$ and south of $60^{\circ} \mathrm{S}$ ), whereas the largest 


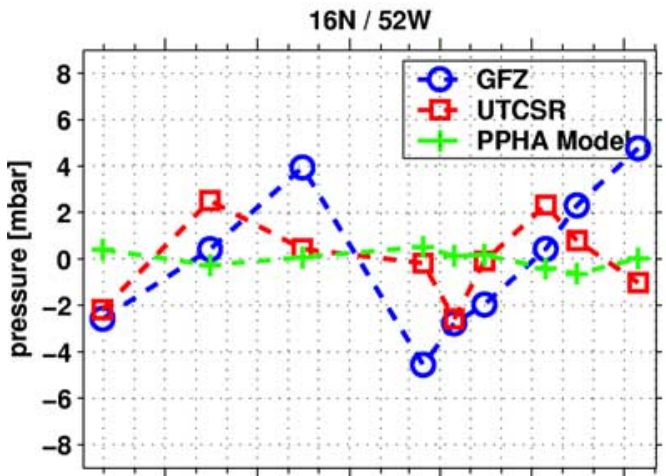

01.Jul 01.Oct 01.Jan 01.Apr 01.Jul 01.Oct 2002

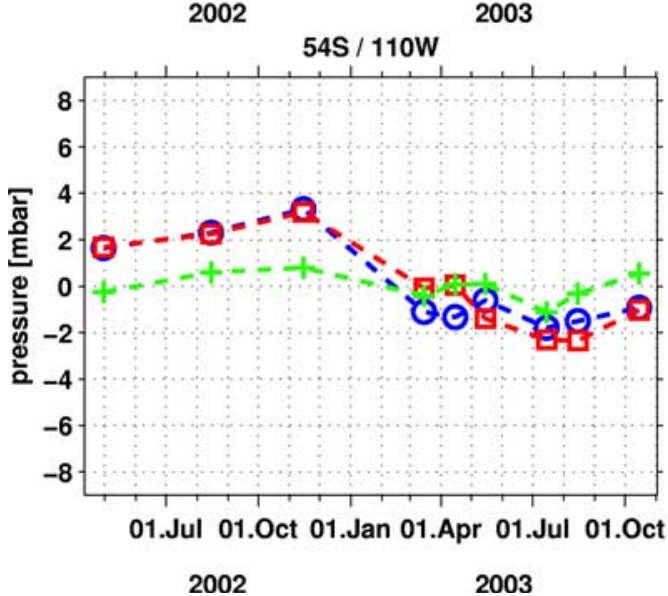

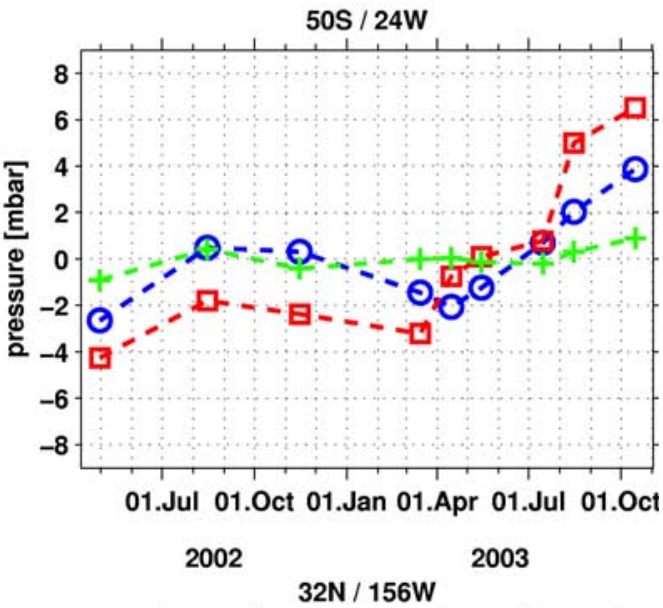

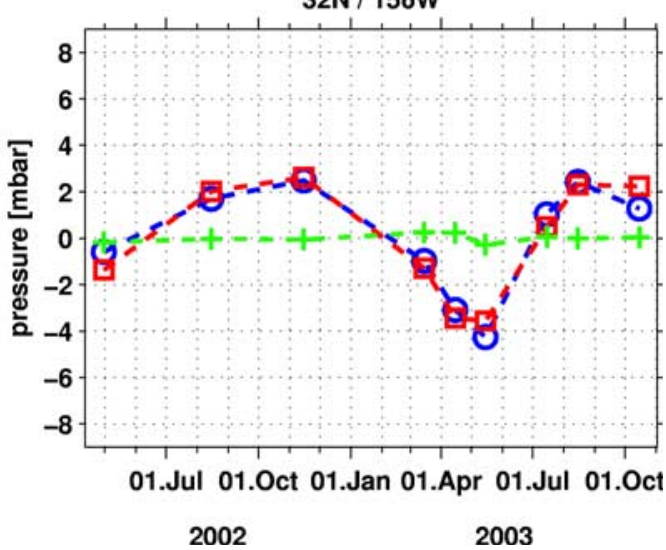

Figure 9. Time series of the OBP at different locations in the world ocean derived from the UTCSR and GFZ monthly gravity field products. For comparison the modeled OBP contribution of the GAC product is also displayed at each location.

deviations are observed at low latitudes, which also corresponds to the findings of V. Zlotnicki (personal communication, 2004). At the same time, the amplitudes of true OBP fluctuations typically decrease toward lower latitudes. As a result, it can be expected that the signal-to-noise ratio of GRACE-derived OBP is better at high latitudes, where the monthly OBP variability reduces to values comparable to those seen in ocean models [Condi and Wunsch, 2004]. The reasons for this are still uncertain, but the convergence of the ground tracks with latitude resulting in improved spatial resolution at the poles may be a candidate. The domain of the PPHA model extends from $65^{\circ} \mathrm{N}$ to $75^{\circ} \mathrm{S}$. Surprisingly, it is near its margins (and beyond) where the most reasonable levels of variability are observed.

[35] Figure 9 displays OBP time series derived from GFZ and UTCSR monthly solutions at four globally distributed locations, along with the GAC monthly value of the signal simulated by the PPHA model that is added back into the original GRACE gravity field solutions. Near the MOVE site (M1, top left) the two gravity products differ in phase and amplitude, with the GFZ solution displaying slightly larger variability. This is in agreement with the large differences seen at this location in the previous comparison (Figure 8, bottom). The significant differences between the GFZ and UTCSR solutions suggest that hydrological leakage from the
Amazon basin contributes comparatively little to the overestimation of GRACE-derived OBP at the MOVE site (as shown in Figure 2), since it would affect either solution in about the same way. However, even with the application of a $1000 \mathrm{~km}$ Gaussian filter, the continental contribution will not vanish completely (see the ellipse with $1000 \mathrm{~km}$ semiaxes drawn around M1). In addition to leakage, the hydrological cycle may also directly affect OBP at M1 through river runoff, and the two error sources are difficult to distinguish.

[36] At a site in the South Atlantic (top right), the UTCSR solution has a consistently larger amplitude than the GFZ one. The phases of the two solutions compare well, which explains why the GFZ-UTCSR differences (Figure 8, bottom) do not display a local maximum at this location. By contrast, at two sites in the Pacific sector (Figure 9, bottom), minimal differences between the products are observed even though the variability in the individual OBP time series is quite strong. As for site M1, the GRACE OBP solutions display unrealistically large variability at the three other locations presented in Figure 9, all of which are relatively free from the influence of hydrological leakage.

[37] It is interesting to note that all of the time series shown in Figure 9 are not dominated by noise on a monthly scale and display real long-term fluctuations which resemble an annual cycle. The monthly contribution from 


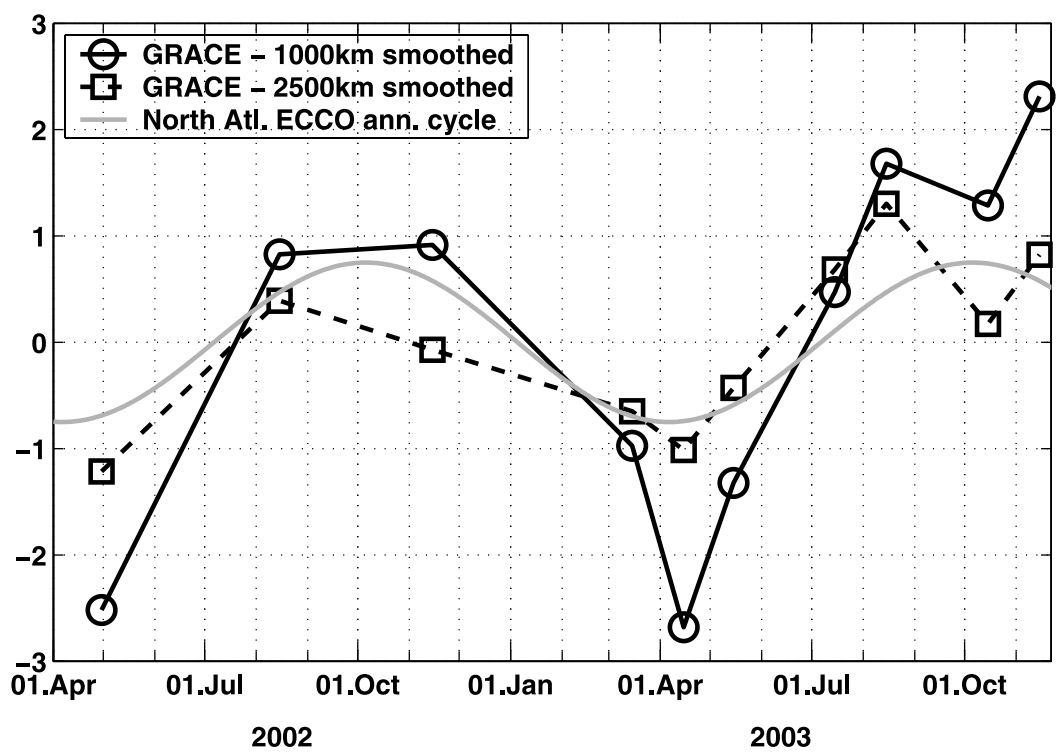

Figure 10. Seasonal evolution of the GRACE OBP signal in mbar averaged over the North Atlantic based on the GFZ monthly gravity field solutions using $1000 \mathrm{~km}$ (circles) and $2500 \mathrm{~km}$ (squares) Gaussian smoothing of the spherical harmonic coefficients. The solid line indicates the annual cycle of the OBP signal from the constrained $1^{\circ} \times 1^{\circ} \mathrm{ECCO}$ model. To reduce possible leakage of the continental hydrological cycle into the ocean, only data from oceanic regions of more than $2000 \mathrm{~m}$ water depth were included. Additionally, OBP has been meridionally averaged between $10^{\circ}$ and $65^{\circ} \mathrm{N}$.

the PPHA model (GAC product) is very small in all cases (typically, less than 1 mbar).

[38] We have discussed the differences between the GRACE products and in situ observations and models. One might wonder whether at this early stage in the data processing, the GRACE-derived OBP fluctuations exhibit realistic spatial patterns. Large spatial-scale variability is qualitatively similar to that from the estimating the circulation and climate of the ocean (ECCO) model [Stammer et $a l ., 2003$ ] in Figure 10. Since at the time the present work was done, the constrained (adjoint) version of the ECCO model did not cover the year 2003, no direct comparison between that model and GRACE products could be carried out. Therefore we extracted the ECCO annual cycle over the North Atlantic for the three year period 2000 to 2002 and applied a harmonic fit to the result. This choice of region was inspired by recent model studies by Condi and Wunsch [2004], who found large areas of coherent phase at the annual cycle in the North Atlantic and North Pacific.

[39] When averaging OBP over the whole North Atlantic, we find reasonable agreement between the phase of the model annual cycle and the seasonal evolution of the GRACE. However, smoothing the GRACE data with a Gaussian filter of $1000 \mathrm{~km}$ half width results in significant overestimation of the amplitude. After applying a $2500 \mathrm{~km}$ filter, much better correspondence in amplitude is obtained. Similar results have been found for the North Pacific [Wenzel et al., 2004]. Thus there is a glimmer of evidence for utility of the GRACE time-varying OBP signal. The measurements may exhibit sensitivity to basin-scale fluctuations of OBP. Interestingly, similar ECCO to GRACE behavior in the South Atlantic cannot be established. This might be attributable to inaccuracies in the ECCO model, because the phase of the annual cycle varies substantially within the South Atlantic, but not in the North Atlantic [Condi and Wunsch, 2004, Figure 9].

\section{Conclusions}

[40] The Earth sciences will definitely benefit from GRACE to a large degree. It provides the most accurate mean and monthly geoid solutions for many applications in geophysics and oceanography. The GRACE time-varying geoid has already successfully been used to detect seasonal hydrological signals over continents [Schmidt et al., 2005], although errors in the preliminary fields are more than an order of magnitude larger than the anticipated GRACE baseline value [Reigber et al., 2005].

[41] The ability of GRACE to recover extremely small time-varying OBP signals must be regarded as one of the greatest challenges of the mission. Over the oceans, the geoid variability is much smaller than over land, and the modeled tidal and nontidal corrections are subject to larger uncertainties. As can be shown from comparisons at the MOVE site, at this stage GRACE still substantially overestimates OBP variability. If instrumental problems (e.g., performance not meeting specifications, nonoptimal processing results from raw data to corrected and calibrated products, or nonoptimal parameterization in gravity field processing) are not the cause, it is most likely that the processing and dealiasing of subinertial and tidal barotropic signals has not been performed with sufficient accuracy. These deficiencies will be further analyzed and improved in coming months by simulation studies (planned at GFZ) or updated background ocean models (a task for the GRACE science team). Nevertheless, GRACE may already possess a marginal capacity to observe ocean dynamics on spatial scales of a few thousand kilometers. 
[42] From a validation study carried out against in situ measurements at the MOVE ground truth site in the tropical northwest Atlantic, apparent deficiencies of the GRACE gravity fields and the models used for dealiasing have been revealed. The atmospherically forced barotropic PPHA model used for nontidal dealiasing agrees reasonably well with in situ observations at periods between 2 and 6 and near 15 days, but significantly underestimates the variance at longer periods. It also appears to underestimate the variability of deep ocean currents in general. It would be desirable to replace it with a full (baroclinic plus more complete barotropic) ocean general circulation model, which has proven to be more consistent with observations over a wider period band. However, the paucity of barotropic data constraints in the ocean limits the accuracy of any numerical model to some degree. At the shortest periods (6 hours), the PPHA model displays much stronger fluctuations than are observed. This could introduce significant aliasing to very long periods. Higher-resolution ECMWF forcing fields may help to overcome this problem. However, such fields are currently not available, and are unlikely to be available in the near future due to inherent sampling limitations.

[43] At the MOVE site, the ocean tide models used for GRACE data processing (FES2002 and CSR4.0) show reasonable overall agreement with each other and with the observations, except for the strongest tidal constituent (M2). Differences in M2 model amplitude of 10 mbar were obtained. The model intercomparison has also revealed that in certain regions, such as equatorial and high latitudes, nonnegligible tide model differences are observed which are explainable by phase discrepancy. It is apparent that the tide models need further improvement. From comparisons at the MOVE site alone, no unequivocal judgment can be made about which of the two tide models is more accurate on a global scale. Additional OBP sites are being maintained in the Atlantic and Pacific sectors, and efforts could be made to more thoroughly verify and improve the models.

[44] OBP derived from the GFZ and UTCSR gravity field products displays similar levels of variability and regional differences in the variability pattern. More importantly, neither product is able to recover the zonally coherent patterns of variability which are robust features of ocean models. Thus the present accuracy of GRACE OBP fluctuations is not sufficient to deduce the evolution of the largescale abyssal flow field. However, it is encouraging to note that time series of GRACE-derived OBP do show longperiod fluctuations, and are not dominated by monthly variability.

[45] Finally, an additional encouraging result ensues from comparison of GRACE with the ECCO model's annual cycle after averaging that signal over the entire North Atlantic. This is the first indication that GRACE might possess some ability to recover the seasonal variability at scales of thousands of kilometers. However, it cannot hold for the longest wavelengths because $\mathrm{C}_{20}$ has been excluded from the analysis.

[46] Constrained ocean model output will become available that covers 2003 (D. Stammer and J. Schröter, personal communication, 2004), and thus will enable more extensive statistical comparisons with the GRACE data. An important issue is the origin of long-period fluctuations of OBP. GRACE OBP signals will likely be more useful at high latitudes where the signal-to-noise ratio is largest for reasons which are presently unclear.

[47] Validation with in situ measurements will remain the ultimate tool to assess the suitability of GRACE gravity fields for oceanic studies. These studies must be extended to other regions. Although in situ observations using point sensors will always lack satisfactory spatial coverage, they are highly accurate and applicable over a broad range of periods extending to years. This, together with the fact that subinertial OBP tends to have a large correlation scale, makes them invaluable. For example, the MOVE ground truth site has been extended for two years starting in January 2004, and additional OBP time series observations carried out at different locations in the Atlantic may soon become available.

[48] Acknowledgments. The German Ministry of Education and Research (BMBF) supports the GRACE Science Data System at GFZ within the Geotechnologien geoscientific $\mathrm{R}+\mathrm{D}$ programme under grant 03F0326A. The authors would like to thank the captains and crews of the research vessels Sonne, l'Atalante and Meteor for their assistance. Support for the MOVE experiment and analysis was provided by the Bundesministerium für Bildung und Forschung within the German CLIVAR programme (grants 03F0246A and 03F0377B) as well as by the Deutsche Forschungsgemeinschaft (grant SE815/21). This work was supported at WHOI by subcontract 1256603 from the Jet Propulsion Laboratory. At the time of revision of this paper, our friend and colleague Peter Schwintzer suddenly passed away. His death is a huge loss, not only for his colleagues, but for the whole scientific community. He is missed greatly.

\section{References}

Bettadpur, S. (2004), UTCSR level-2 processing standards document for product release 01, GRACE Proj. Doc. JPL 327-742, rev. 1.1, Jet Propul. Lab., Pasadena, Calif.

Condi, F., and C. Wunsch (2004), Measuring gravity field variability, the geoid, ocean bottom pressure fluctuations, and their dynamical implications, J. Geophys. Res., 109, C02013, doi:10.1029/2002JC001727.

Flechtner, F. (2003a), GFZ level-2 processing standards document for product release 01, GRACE Proj. Doc. JPL 327-743, rev. 1.0, Jet Propul. Lab., Pasadena, Calif.

Flechtner, F. (2003b), AOD1B product description document, GRACE Proj. Doc. JPL 327-750, rev. 1.0, Jet Propul. Lab., Pasadena, Calif.

Han, S.-C., C. Jekeli, and C. K. Shum (2004), Time-variable aliasing effects of ocean tides, atmosphere, and continental water mass on monthly mean GRACE gravity field, J. Geophys. Res., 109, B04403, doi:10.1029/ 2003JB002501.

Hirose, N., I. Fukumori, V. Zlotnicki, and R. Ponte (2001), High-frequency barotropic response to atmospheric disturbances: Sensitivity to forcing, topography, and friction, J. Geophys. Res., 106, 30,987-30,995.

Hughes, C. W., C. Wunsch, and V. Zlotnicki (2000), Satellite peers through the oceans from space, Eos Trans. $A G U, 81(7), 68$.

Kamenkovich, I. V., and J. Pedlosky (1998a), Radiation of energy from nonzonal ocean currents, nonlinear regime. part I: Single wave development, J. Phys. Oceanogr., 28, 1661-1682.

Kamenkovich, I. V., and J. Pedlosky (1998b), Radiation of energy from nonzonal ocean currents, nonlinear regime. part II: Interactions between waves, J. Phys. Oceanogr., 28, 1683-1701.

Kanzow, T. (2000), Integrale Erfassung langperiodischer Transporte: Simulation und Optimierung eines verankerten Systems, Diplomarbeit, Inst. für Meereskunde, Univ. Kiel, Kiel, Germany.

Kanzow, T. (2004), Monitoring the integrated deep meridional flow in the tropical North Atlantic, dissertation, Christian-Albrechts-Univ. zu Kiel, Kiel, Germany.

Knudsen, P. (2003), Ocean tides in GRACE monthly averaged gravity fields, Space Sci. Rev., 108(1-2), 261-270.

LeProvost, C. (2002), FES2002-A new version of the FES tidal solution series, paper presented at Jason-1 Science Working Team Meeting, Cent. Natl. d'Etudes Spatiales, Biarritz, France.

Lohmann, K. (1999), Gezeitenkorrektur von schiffsgestützten Strömungsmessungen, Diplomarbeit, Inst. für Meereskunde, Univ. Kiel, Kiel, Germany.

Luther, D., and A. Chave (1993), Observing integrating variables in the ocean, in Proceedings 7th Aha Huliko'a Hawaiian Winter Workshop on Statistical Methods in Physical Oceanography, edited by P. Muller and D. Henderson, pp. 103-128, Univ. of Hawaii, Honolulu. 
Miller, A. J., W. R. Holland, and M. C. Hendershott (1987), Openocean response and normal mode excitation in an eddy-resolving general circulation model, Geophys. Astrophys. Fluid Dyn., 37, $253-278$.

Pedlosky, J. (1987), Geophysical Fluid Dynamics, 624 pp., Springer, New York.

Philander, S. (1978), Forced oceanic waves, Rev. Geophys., 16, 15-46.

Ponte, R. (1993), Variability in a homogeneous global ocean forced by barometric pressure, Dyn. Atmos. Oceans, 18, 209-234.

Ponte, R., and R. Ray (2002), Atmospheric pressure corrections in geodesy and oceanography: A strategy for handling tides, Geophys. Res. Lett., 29(24), 2153, doi:10.1029/2002GL016340.

Reigber, C., R. Schmidt, F. Flechtner, R. König, U. Meyer, K.-H Neumayer, P. Schwintzer, and S. Y. Zhu (2005), An Earth gravity field model complete to degree and order 150 from GRACE: EIGENGRACE02S, J. Geodyn., 39, doi:10.1016/j.jog.2004.07.00.

Schmidt, R., et al. (2005), GRACE observations of changes in continental water storage, Global Planet. Change, in press.

Send, U., T. Kanzow, W. Zenk, and M. Rhein (2002), Monitoring the Atlantic meridional overturning circulation at $16^{\circ} \mathrm{N}$, CLIVAR Exchanges, $7,31-33$.

Song, Y., and V. Zlotnicki (2004), Ocean bottom pressure waves predicted in the tropical Pacific, Geophys. Res. Lett., 31, L05306, doi:10.1029/ 2003GL018980.

Stammer, D., C. Wunsch, R. Giering, C. Eckert, P. Heimbach, J. Marotzke, A. Adcroft, C. N. Hill, and J. Marshall (2003), Volume, heat, and freshwater transports of the global ocean circulation 1993-2000, estimated from a general circulation model constrained by World Ocean Circulation Experiment (WOCE) data, J. Geophys. Res., 108(C1), 3007, doi:10.1029/ 2001JC001115.

Tapley, B., and C. Reigber (2004), GRACE (Gravity Recovery and Climate Experiment), in McGraw-Hill 2004 Yearbook of Science and Technology, pp. 135-138, McGraw-Hill, New York.
Tapley, B., S. Bettadpur, J. Ries, P. Thompson, and M. Watkins (2004), GRACE measurements of mass variability in the Earth system, Science, 305, 503-505, doi:10.1126/science.1099192.

Wahr, J., S. Jayne, and F. Bryan (2002), A method of inferring changes in deep ocean currents from satellite measurements of time-variable gravity, J. Geophys. Res., 107(C12), 3218, doi:10.1029/2001JC001274.

Wahr, J., S. Swenson, V. Zlotnicki, and I. Velicogna (2004), Time-variable gravity from GRACE: First results, Geophys. Res. Lett., 31, L11501, doi:10.1029/2004GL019779.

Wenzel, M., F. Flechtner, R. Schmidt, C. Reigber, V. Seufer, B. Fritzsch, and J. Schröter (2004), Verification of an ocean general circulation model with time varying GRACE geoid data, paper presented at the Joint Champ/GRACE Science Meeting, GeoForschungsZentrum Potsdam, Potsdam, Germany, 6-8 July.

Wiehl, M., and R. Dietrich (2005), Time-variable gravity seen by satellite missions: On its sampling and its parametrization, in Earth Observation With CHAMP-Results From Three Years in Orbit, edited by C. Reigber et al., pp. 121-126, Springer, New York.

Wunsch, C. (1997), The vertical partition of oceanic horizontal kinetic energy, J. Phys. Oceanogr., 27, 1770-1794.

A. Chave, Woods Hole Oceanographic Institution, Woods Hole, MA 02543, USA.

F. Flechtner and R. Schmidt, Department 1 (Geodesy and Remote Sensing), GeoForschungsZentrum Potsdam (GFZ), Telegrafenberg A17, D-14473 Potsdam, Germany. (flechtne@gfz-potsdam.de)

T. Kanzow and U. Send, Leibniz-Institut für Meereswissenschaften an der Universität Kiel (IFM-GEOMAR), Düsternbrooker Weg 20, D-24105 Kiel, Germany. 